\title{
Pulse-Inversion Chirp-Coded Weld Harmonic Imaging (PI-CWHI) of Friction Stir Welded Butt-Joint
}

\author{
M. Tabatabaeipour ${ }^{1}$ - J. Hettler ${ }^{1}$ - N. T. Sewell ${ }^{2}$ - J. R. Wright ${ }^{2}$ - J. C. S. Wright ${ }^{2}$. \\ S. Delrue ${ }^{1}$ - K. Van Den Abeele ${ }^{1}$
}

Received: 2 July 2016 / Accepted: 31 July 2017

(C) Springer Science+Business Media, LLC 2017

\begin{abstract}
The microscopic size and the closed characteristics of zigzag line defects at the root of friction stir welded joints frequently result in a detection inability by off-theshelf ultrasonic weld inspection techniques. To overcome this problem, we take advantage of a non-linear ultrasonic approach by employing a slanted contact pitch-catch mode scanning using chirp-coded excitation with subsequent pulse-inversion analysis. We validated that the observed nonlinearity correlates well with the micro-crack density along the weld path. Doing so, hot spots in the pulse-inversion chirp-coded weld harmonic images can be interpreted as zones with high concentrations of micro-flaws.
\end{abstract}

Keywords Friction stir welding - Micro-crack density · Zigzag line defects - Continuous oxide film · Pulseinversion - Chirp-coded excitation · Harmonic imaging · Non-destructive testing

\section{Introduction}

\subsection{Friction Stir Welding}

Friction stir welding (FSW) was first introduced in 1991 as a solid-state joining technique by The Welding Institute (TWI-UK), and is nowadays used for a wide range of industrial applications in the field of aerospace, automotive and shipbuilding construction [1,2]. The FSW joining pro-

\footnotetext{
M. Tabatabaeipour

s.m.tabatabaeipour@gmail.com

1 Wave propagation and Signal Processing, Department of Physics, KU Leuven Kulak, 8500 Kortrijk, Belgium

2 Theta Technologies Limited, Exeter EX4 4RN, UK
}

cedure is considered to be an environmentally friendly and energy-efficient method. Nevertheless, FS welded joints are known to be susceptible to a variety of welding flaws such as lack of penetration (LOP), wormholes, kissing bonds and micro-cavities accompanying a zigzag line (also known as micro-cracked zigzag line). Micro-cracks and kissing bonds (potentially caused by the presence of a trapped continuous oxide film from the initial butt surfaces) are particular discontinuities whose adjacent surfaces are in close contact with each other, however, with little or no metallic bonding. The difference between them is that kissing bonds are generally linked to the butt surface and therefore will adversely affect the bending property in the root region of the welded joint [3]. Note that kissing bond defects are also utterly different from LOP which may occur at the butt surface of the welds [4]. While LOP defects mainly occur when both sides of the root region are not properly forged, kissing bonds originate at the root of the weld because of insufficient plunging of the welding tool during friction stir welding and/or low heat input due to an inappropriate setting of the welding procedure parameters such as rotational and transversal speeds. The effects of the welding parameters on the formation of kissing bonds and zigzag lines in the root part of the weld have been investigated by Sato et al [3]. They showed that the presence of a continuous oxide film may initiate cracking along a zigzag line during root bending. Furthermore, they found that micro-cavities accompanying the zigzag line are likely to happen at the top, bottom or across the nugget zone. Similarly, Dai et al. reported the occurrence of micro-cracks or cavities along the zigzag line depending on heat input conditions. They also observed that these imperfections significantly affect the face bend properties of the FSW joint with limited effect on the tensile properties [5]. Several other studies support the fact that the fatigue life and strength of the FSW joint is significantly impaired by the presence of 
kissing bonds and zigzag line defects at the root part of the weld $[3,6,7]$.

To counteract the problem of reduced fatigue life of welds due to imperfections, conventional non-destructive testing techniques need to be revised and upgraded to enable the detection of micro-defects before they become disastrous. Many efforts along this line have been proposed in recent years. For instance, using eddy current technology, Goldfine et al. have demonstrated the capability of multi-element eddy current sensors to inspect the FSW from the root side of the sample by measuring absolute electrical conductivity $[8,9]$. Lately, Tabatabaeipour et al. suggested an optimized linear ultrasonic method, employing immersion ultrasonic pulseecho testing from the tool side of the samples with empirical positioning and time gating [4]. In both ultrasonic and eddy current methods, B-scan (transverse section) and C-scan (top view) profiles can be obtained allowing an easy (and operator independent) interpretation of the different microstructural regions within the friction stir welded zone as well as the presence of (sizeable) linear defects at the root of the nugget zone. An important point to note here is that these techniques only reveal information about linear defects and/or linear segment of defects, such as "open" cracks or crack-lips with stress-free surfaces. Nonlinear defects such as micro-cracked zigzag line defects do not necessary exhibit elastic discontinuities at their crack faces. That is why linear techniques generally cannot identify them. The presence of such defects can be inspected by advanced nonlinear ultrasonic techniques, as explained in more detail in the following section.

\subsection{Pulse-Inversion Technique}

Recent experimental and simulation studies have demonstrated the potential of nonlinear ultrasonic techniques for nondestructive testing and evaluation and their application to a wide variety of materials containing diverse types of defects. In brief, nonlinear techniques investigate the violation of the linear superposition principle of waves by quantifying the generation of sub-harmonics [10], second and higher harmonics [11], the loss of proportionality in the response [12], or the amplitude dependent resonance frequency shift [13]. One of the primary indicators of nonlinearity used in many investigations is the generation of higher harmonic components in an ultrasonic signal when propagating through a material containing defects. Since harmonic components are much weaker than the fundamental, techniques have been proposed to enhance their presence as much as possible relative to noise. For instance, to isolate and intensify the second harmonic in the signal, multi-pulse practices such as the so-called Pulse-Inversion (PI) technique have been commonly applied in recent years as a nonlinearity enhancing method $[14,15]$. In the PI technique, two consecutive excitations are considered for each acoustic beam line. After a first pulse is transmitted, the excitation pulse is inverted and transmitted again during a second firing. By summing the received signals, the contribution of the fundamental frequency (linear part of the signal) will be automatically suppressed, due to the phase inversion. In the case of a linear system, the summed signal will thus only contain noise. In case of a nonlinear system, the fundamental signal, as well as all odd harmonics, are again eliminated because of their out of phase components, while the even harmonic components in the summed signal will be doubled in amplitude. The net result is a non-zero output offering an enhanced indication of the nonlinearity [16-19].

Mathematically, the response to a given excitation $\boldsymbol{x}(\boldsymbol{t})$ with amplitude $\boldsymbol{A}_{1}$ in a simple nonlinear system, supposing a zero-memory nonlinear constitutive model, can be expressed as [20]:

$$
R_{0}=A R_{1}(t)+A^{2} R_{2}(t)+A^{3} R_{3}(t)+\ldots
$$

where $\boldsymbol{A}$ is proportional to $\boldsymbol{A}_{1}$, and $\boldsymbol{A} \boldsymbol{R}_{1}(\boldsymbol{t}), \boldsymbol{A}^{\mathbf{2}} \boldsymbol{R}_{2}(\boldsymbol{t})$ and $A^{\mathbf{3}} \boldsymbol{R}_{\mathbf{3}}(t)$ stand for the linear signal response, and the contribution to the subsequent harmonic components respectively.

In the pulse-inversion approach, the second excitation waveform changes to negative polarity, i.e. $\boldsymbol{A}_{\mathbf{2}}=-\boldsymbol{A}_{\mathbf{1}}$. Therefore, the response to the negative polarity (out of phase) excitation can be written as:

$$
R_{180}=-A R_{1}(t)+A^{2} R_{2}(t)-A^{3} R_{3}(t)+\ldots
$$

When summing Eqs. (1) and (2)

$R_{P I}=R_{0}+R_{180}=2 A^{2} R_{2}(t)+\ldots$,

only the even order harmonics with double amplitude are retained, while the noise power will decrease by a factor of $\sqrt{ } \mathbf{2}$, leading to a substantial increase in the signal to noise ratio (SNR). Therefore, the PI technique plays an effective role in improving the harmonic image quality.

For the FSW inspection of micro-cracked zigzag line defects, we will consider a linear chirp-coded excitation, followed by the pulse-inversion analysis. A linear chirp is a coded signal that linearly spans over a frequency bandwidth $B=f_{2}-f_{1}$, where $f_{1}$ and $f_{2}$ are the starting and ending frequencies respectively over a time $\boldsymbol{T}$. The time history of a linear chirp can be expressed as follows:

$s(t)=w_{0}(t) e^{j 2 \pi\left(f_{1}+\frac{\mu}{2} t\right) t}$

where the $\boldsymbol{w}_{\mathbf{0}}(\boldsymbol{t})$ is a window function (also known as apodization function), and $\boldsymbol{\mu}=\frac{\boldsymbol{B}}{T}$ is the constant rate of frequency change during the time interval $\mathrm{T}$.

There are two main reasons to favor a chirp-coded excitation. First, the transmitted energy, and consequently the 
penetration depth and the SNR, can be substantially (15$20 \mathrm{~dB}$ ) increased by coded excitation techniques. Coded excitation can be categorized in "phase" coding, as in the bi-phase Barker and Golay, and "frequency" coding, as in a chirp signal [21-23]. The most common frequency code excitation is the linear chirp used here for FSW inspection. Chiao et al. have shown that Golay and chirp signals have a similar peak range sidelobe level (PRSL). In addition, Shekhar et al. investigated the effects of the transmit parameters on the nonlinearity response, and demonstrated an improvement of the SNR in the high frequency harmonic imaging when a chirp-coded excitation is used rather than a sine-burst excitation [24]. Furthermore, as coded excitation may suffer from an increased sidelobe artifact due to the potential spectral overlap between the fundamental and the harmonic components of the received signal, Song et al. evaluated the coded harmonic imaging technique using three different harmonic extraction techniques: bandpass filtering $(\mathrm{BF})$, power modulation $(\mathrm{PM})$ and pulse-inversion (PI) [25]. Their simulations and in-vitro experiments show that the pulse-inversion based coded harmonic imaging method

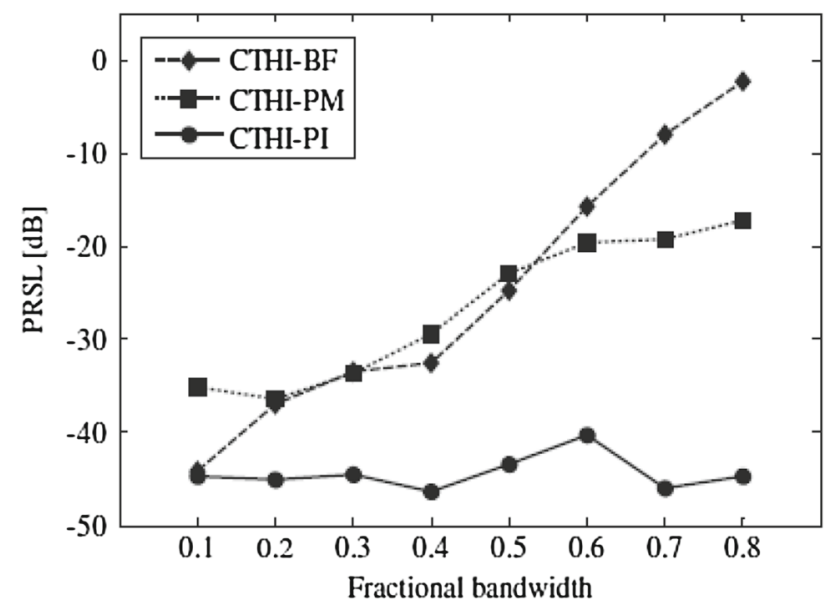

Fig. 1 Peak range sidelobe level (PRSLL) of three different harmonic extraction methods ( $B F$ bandpass filtering, $P M$ power modulation, $P I$ pulse inversion) as a function of the fractional bandwidth of the transmitted chirp-coded signal, illustrating consistent results of the pulse-inversion harmonic extraction technique, photo courtesy of [25] outperforms the other extraction methods due to its excellent cancellation of the fundamental and the third harmonic components, and its adequate suppression of the spectral leakage (range sidelobe artifacts), as illustrated in Fig. 1. As a result, it produces a better image contrast. Likewise, Park et al. reported a $34 \%$ enhancement of the contrast to noise ratio of the pulse-inversion chirp-coded harmonic imaging over conventional pulse harmonic imaging, as well as a reduction of $11 \mathrm{~dB}$ in the sidelobe level of the pulseinversion chirp coded harmonic with respect to the level obtained in bandpass filtered chirp coded harmonic imaging [26].

A second reason for using chirp-coded excitation, rather than sine-burst excitation, is that it enhances the likelihood of hitting a particular micro-crack resonance frequency, which subsequently increases the generation of higher harmonics $[27,28]$. In other words, when using a chirp-coded excitation with a long duration, the nonlinear response will be activated, and potentially detected, for a wider range of micro-cracks.

\section{Experimental Setup}

A contact pitch-catch experiment, with a set-up as schematically shown in Figure 2, was conducted in order to validate the use of the pulse-inversion technique for the inspection of micro-crack concentrations in a friction stir welded buttjoint. Two transducers, one being a transmitter and the other one a receiver, were mounted on rexolite wedges in order to generate shear waves in the welded sample. The transducers were employed in pitch-catch mode, aligned and separated to maximize the reflection from the bottom of the sample.

The weld samples in this study (kindly provided by TWI) are made of aluminum alloy, 7XXX series, with $6.35 \mathrm{~mm}$ thickness. Two large test samples (dimensions $500 \times 300 \times 6.35 \mathrm{~mm}$ ) for which results will be discussed here, were manufactured under imperfect welding conditions to deliberately initiate root flaws along at least part of the weld length. The weld zone running up the center is about $25 \mathrm{~mm}$ wide and $500 \mathrm{~mm}$ long.
Fig. 2 Schematic representation of the contact pitch-catch method for the inspection of friction stir welded butt-joint from the tool side, $\mathrm{T}$ and $\mathrm{R}$ stand for transmitter and receiver respectively

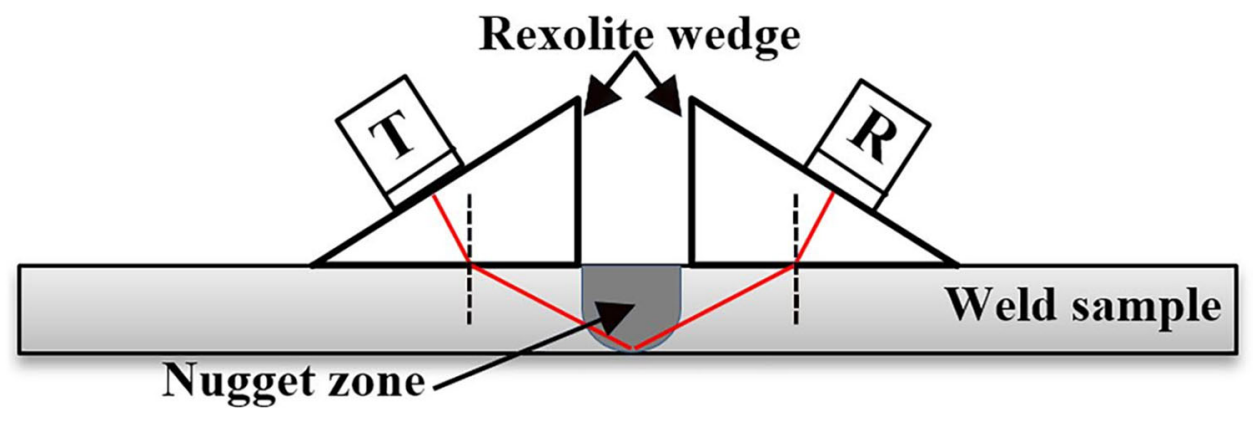




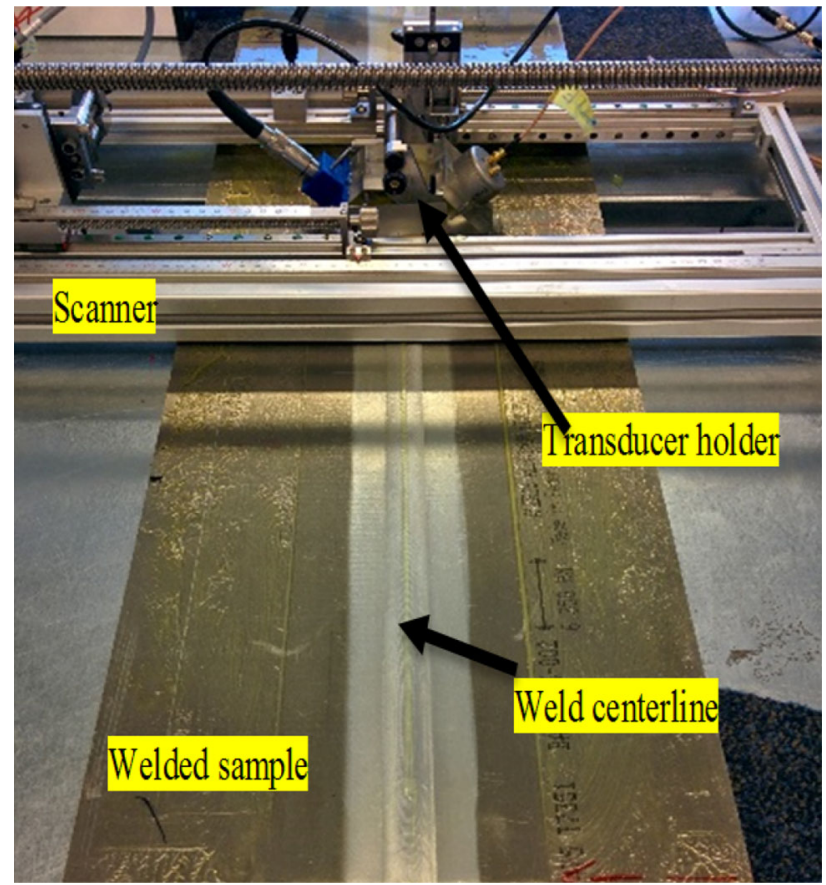

Fig. 3 Inspection set-up with the transducer head and the scanner that allows to move along the weld centerline
The measurements were carried out using an arbitrary waveform generator and a data acquisition card, controlled by a PC. An r.f. amplifier was used for the input signal amplification. In order to examine the samples along the weld centerline, a flat scanner was designed and manufactured. The manual X-Y scanner, with position feedback, was used for the scan along the $500 \mathrm{~mm}$ long weld centerline, see Fig. 3.

\section{Methodology}

The excitation signals provided to the transmitter consist of two consecutive out of phase chirp signals ( 0 phase and 180 phase) with frequencies linearly ramping up from 2 to $5 \mathrm{MHz}$ (fractional bandwidth of 0.85) as shown in Fig. 4. The two chirp signals are transmitted across the weld at each position along the scanline. Figure 5 illustrates the typically received response signals ( $R_{0}$ for 0 phase or positive polarity, and $R_{180}$ for 180 phase or negative polarity), while the amplitude spectrum of the received positive polarity response $\left(R_{0}\right)$ is displayed in Fig. 6, confirming that the main frequency band of the response ranges from 2 to $5 \mathrm{MHz}$.

Subsequently, the two opposite-phase back-wall reflections are summed to obtain the pulse-inversion signal. The
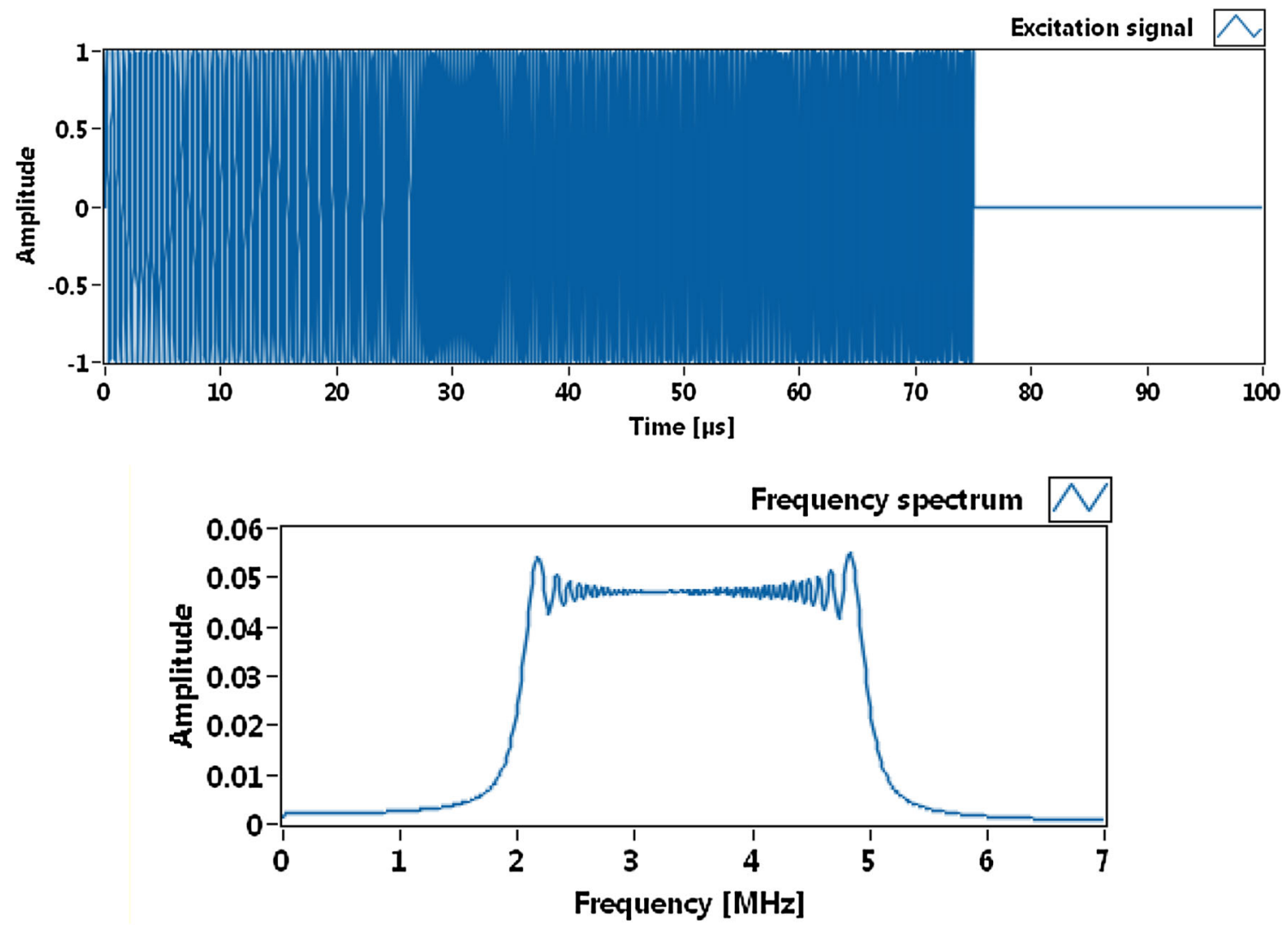

Fig. 4 Excitation chirp signal with 0 phase, ranging from 2 to $5 \mathrm{MHz}$ with a $75 \mu$ s length. Top time domain, Bottom frequency domain 

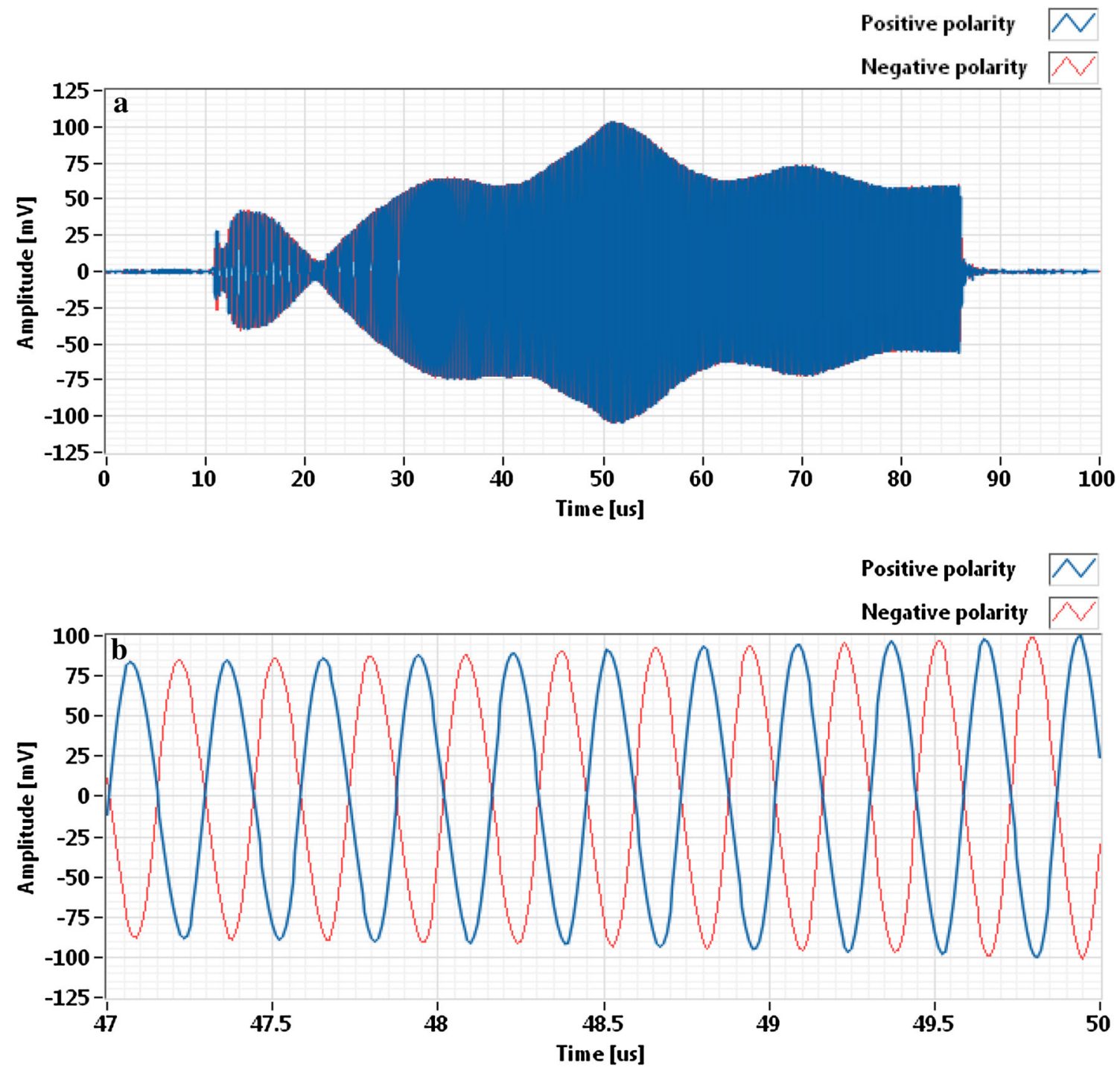

Fig. 5 a Overlay of typical signal responses to two out of phase chirp-coded excitation signals, b zoomed-in representation

Fig. 6 Frequency spectrum of the positive polarity response, showing a fundamental frequency band ranging from 2 to $5 \mathrm{MHz}$ around the center frequency of $3.5 \mathrm{MHz}$, yielding a 0.85 fractional bandwidth

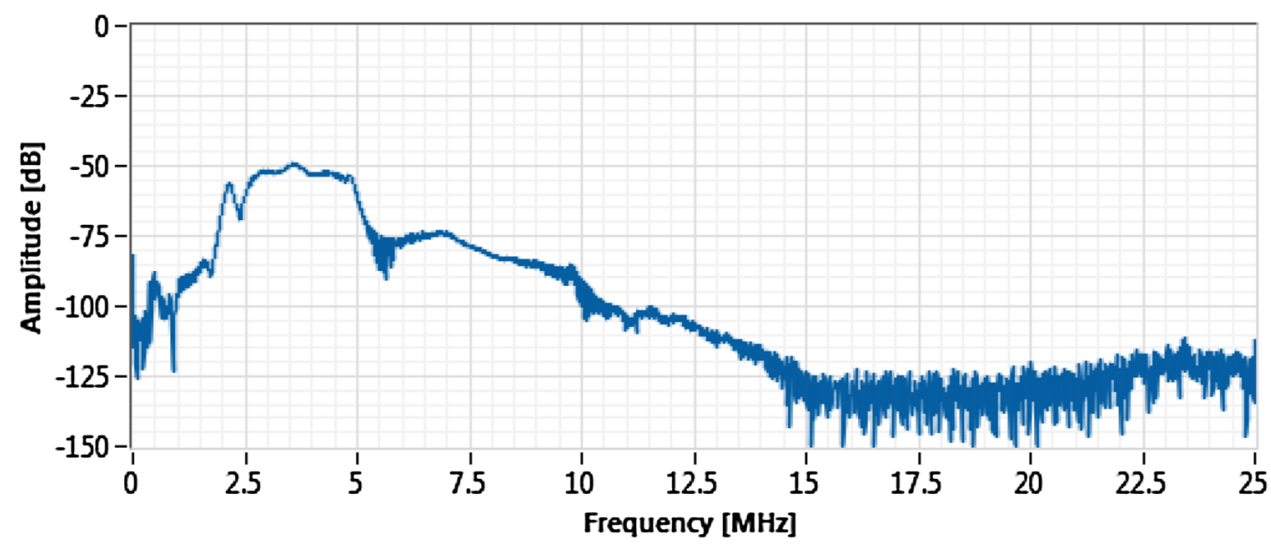


Fig. 7 Normalized pulse inversion spectrum (NPIS) of the pulse-inversion signal illustrating that the second harmonic content is $35 \mathrm{~dB}$ higher than the peak sidelobe level

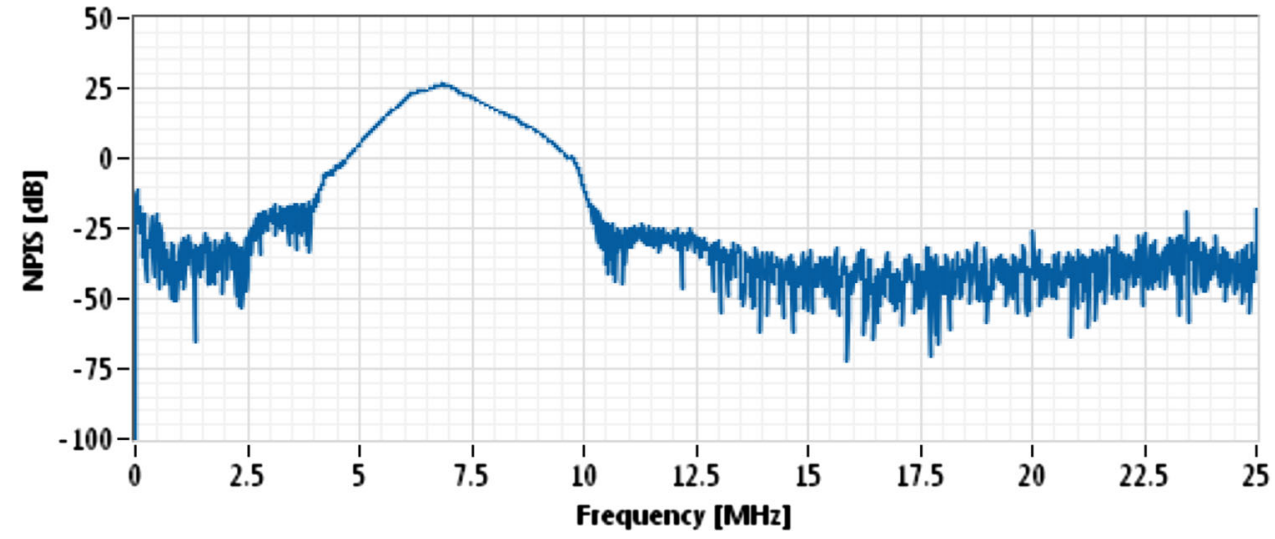

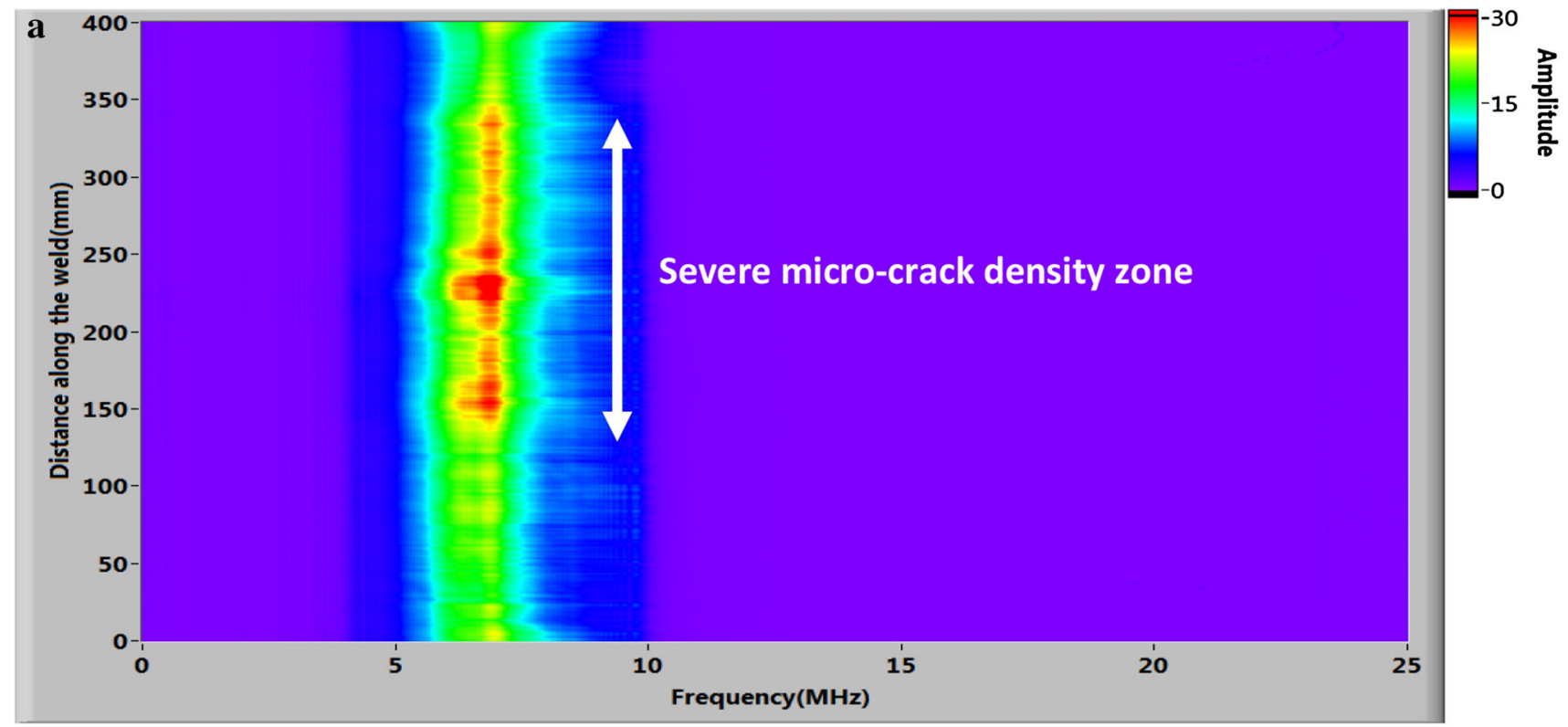

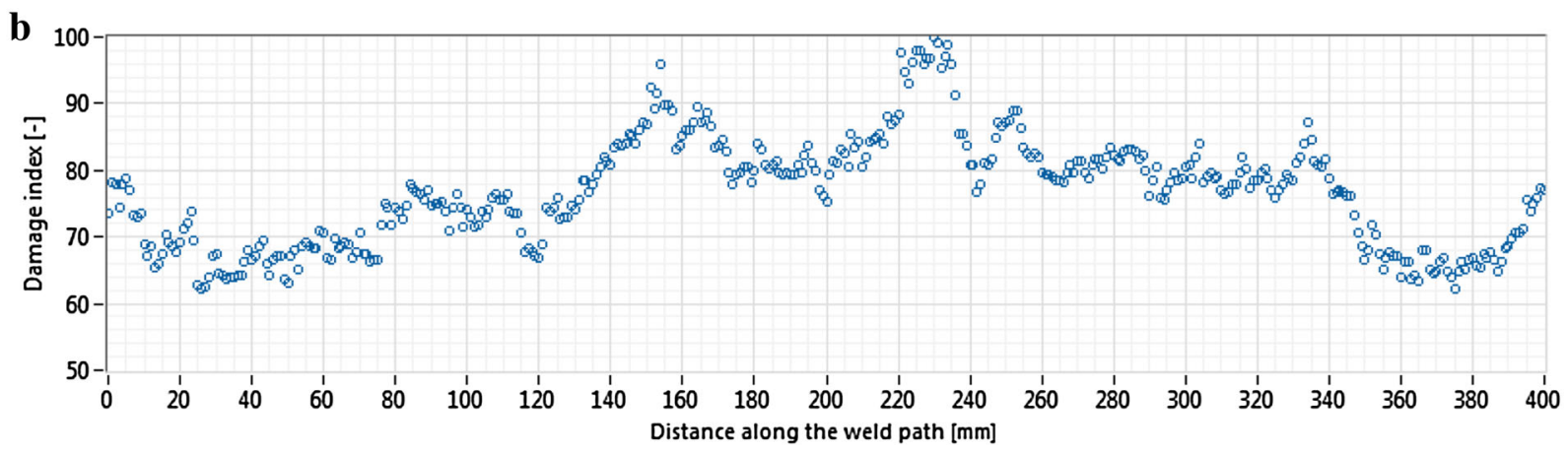

Fig. 8 a Pulse-inversion chirp-coded weld harmonic image obtained by stacking the normalized frequency spectra for each position along the weld centerline, indicating a zone of severe micro-crack density between 150 and $350 \mathrm{~mm}$, Sample1. b Damage Index Scan along the weld center line, according to Eq. (6)

normalized frequency spectrum of the pulse-inversion signal is then calculated as follows:

$N P I S=\frac{F F T\left(R_{0}+R_{180}\right)}{F F T\left(R_{0}\right)_{\max }^{2}} \propto \frac{A_{2}}{A_{1}^{2}}$ where $\boldsymbol{R}_{\mathbf{0}}$ and $\boldsymbol{R}_{\mathbf{1 8 0}}$ are the 0 phase and the 180 phase response signals respectively, and $N \boldsymbol{P} I S$ stands for the Normalized Pulse-Inversion Spectrum. $\boldsymbol{A}_{\mathbf{2}}$ and $\boldsymbol{A}_{\mathbf{1}}$ are representative for the second harmonic and fundamental amplitudes respectively. By taking the ratio of $\boldsymbol{A}_{\mathbf{2}}$ to the square of $\boldsymbol{A}_{\mathbf{1}}$, the 

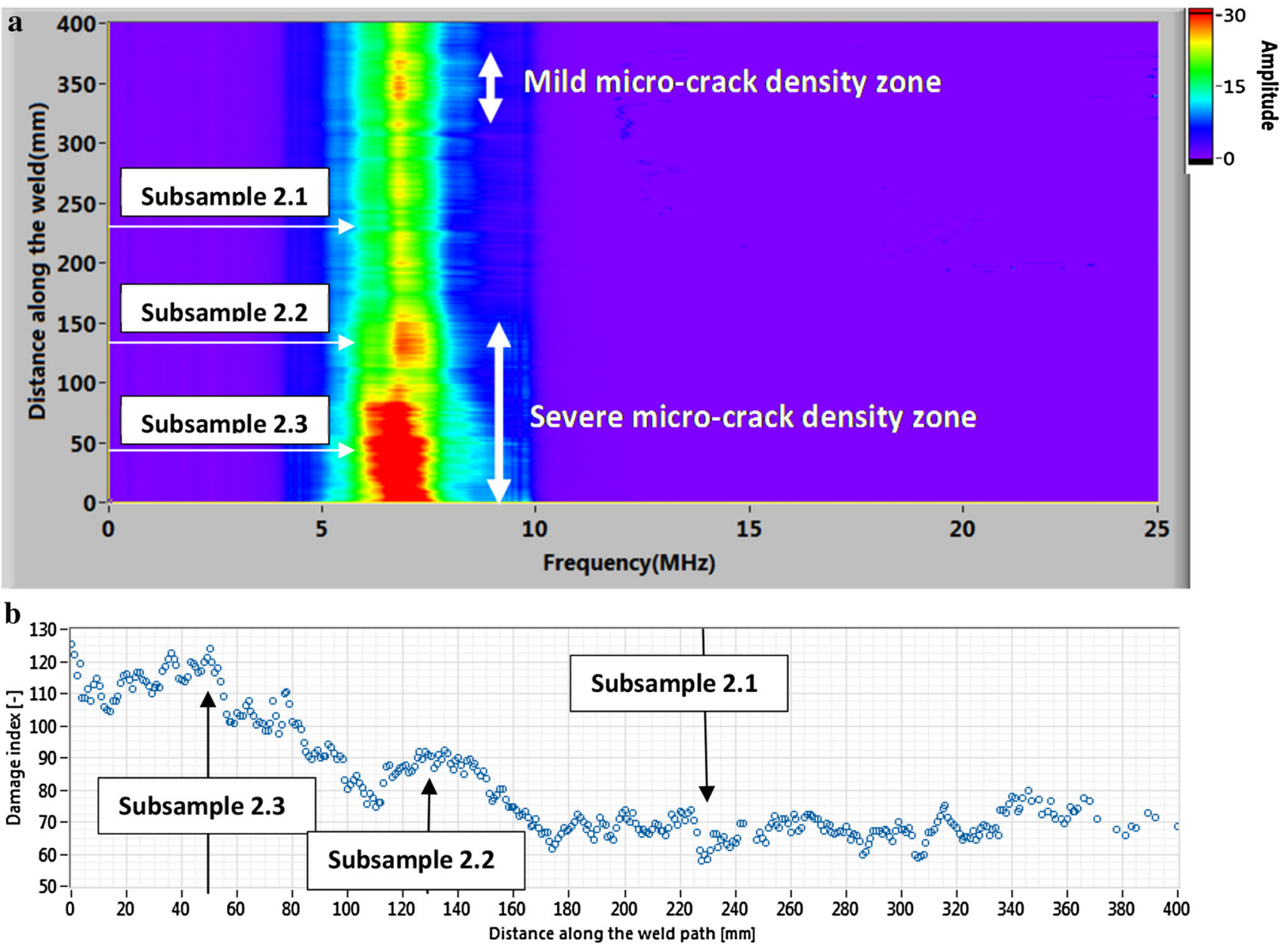

Fig. 9 a Pulse-inversion chirp-coded weld harmonic imaging, indicating a zone of severe micro-crack density at the beginning of the weld path, and additional sites of mild micro-crack density, Sample2. b Dam- age Index Scan along the weld center line, according to Eq. (6). The horizontal arrows point to the position along the weldline for which subsamples are tested using optical microscopy and SEM

Table 1 Results of the number of micro-cracks observed in the optical microscopy

\begin{tabular}{lclc}
\hline Subsample & Damage index & Number of micro-cracks & Area of micro-cracks $\left(\mathrm{mm}^{2}\right)$ \\
\hline Low level (subsample 2.1) & 60 & 2 & 0.0015 \\
Average level (subsample 2.2) & 90 & 5 & 0.0035 \\
High level (subsample 2.3) & 120 & 20 & 0.015 \\
\hline
\end{tabular}

quotient $\boldsymbol{N} \boldsymbol{P} \boldsymbol{I} \boldsymbol{S}$ represents a signature of nonlinearity [29]. As can be observed in Fig. 7, the fundamental frequency components have been completely canceled out by the PI technique. The principal frequency components in the PI signal span the region between 4 to $10 \mathrm{MHz}$. As the generation of harmonics is linked to the presence of damage, the NPIS can be used as a (frequency dependent) damage indicator (zero corresponding to total absence of nonlinearity/damage). A scalar damage index can be obtained by integrating the NPIS over the relevant frequency band. For instance,
$D I_{N P I S}=\int_{4 M H z}^{10 M H z}\left|\frac{F F T\left(R_{0}+R_{180}\right)}{F F T\left(R_{0}\right)_{\max }^{2}}\right| d f$

We expect this damage index to consequently increase when the micro-crack density increases.

\section{Results and Discussion of Harmonic Imaging}

The above described frequency spectrum analysis, employing equation (5), was applied to all pairs of A-scan signals 

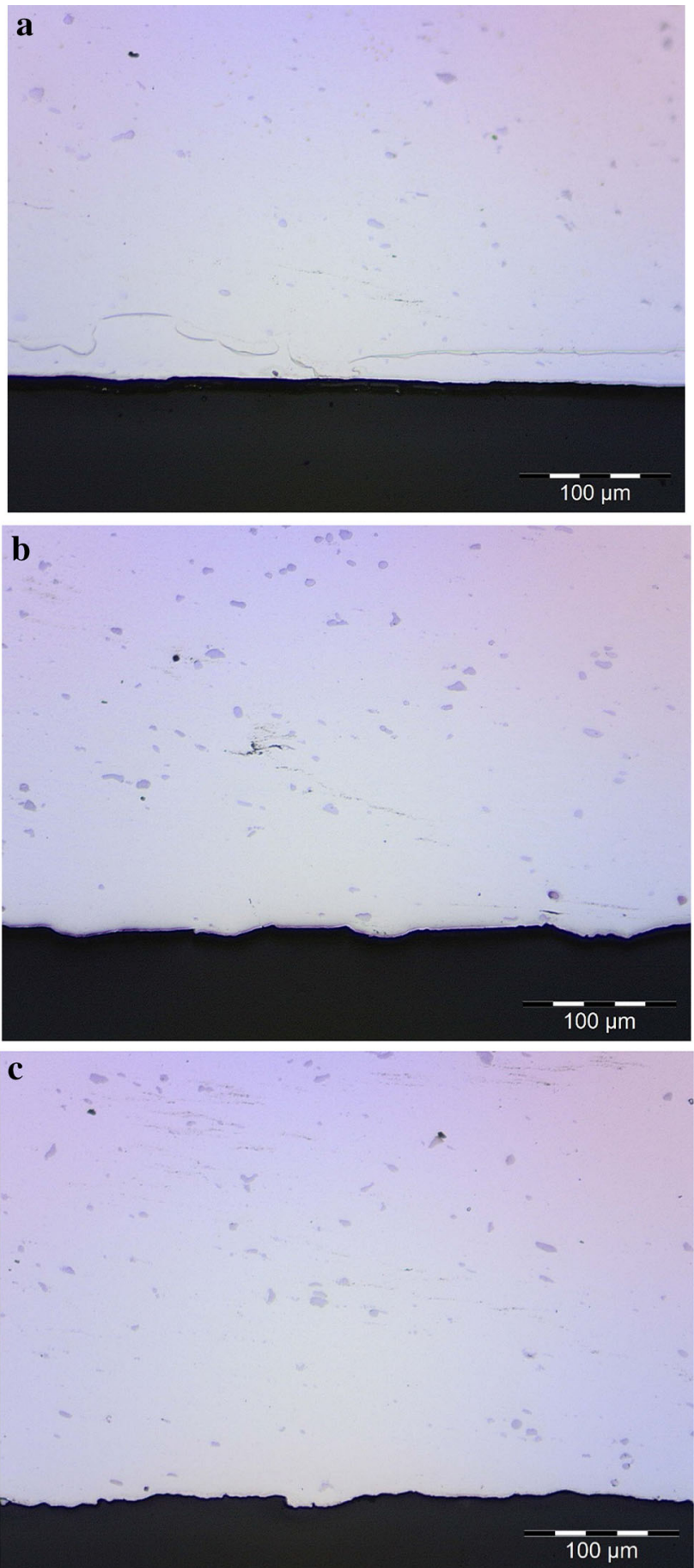

Fig. 10 Cross-sectional view of three subsamples from FSW Sample 2, obtained by optical microscopy. Level of nonlinearity obtained from PI-CWHI a low level at $230 \mathrm{~mm}$, b average level at $130 \mathrm{~mm}$, c high level at $50 \mathrm{~mm}$, after [30]

acquired along the weld centerline. A spectral image was then produced in a pseudo-color plot by stacking up all NPIS data as function of the position along the weld. As shown
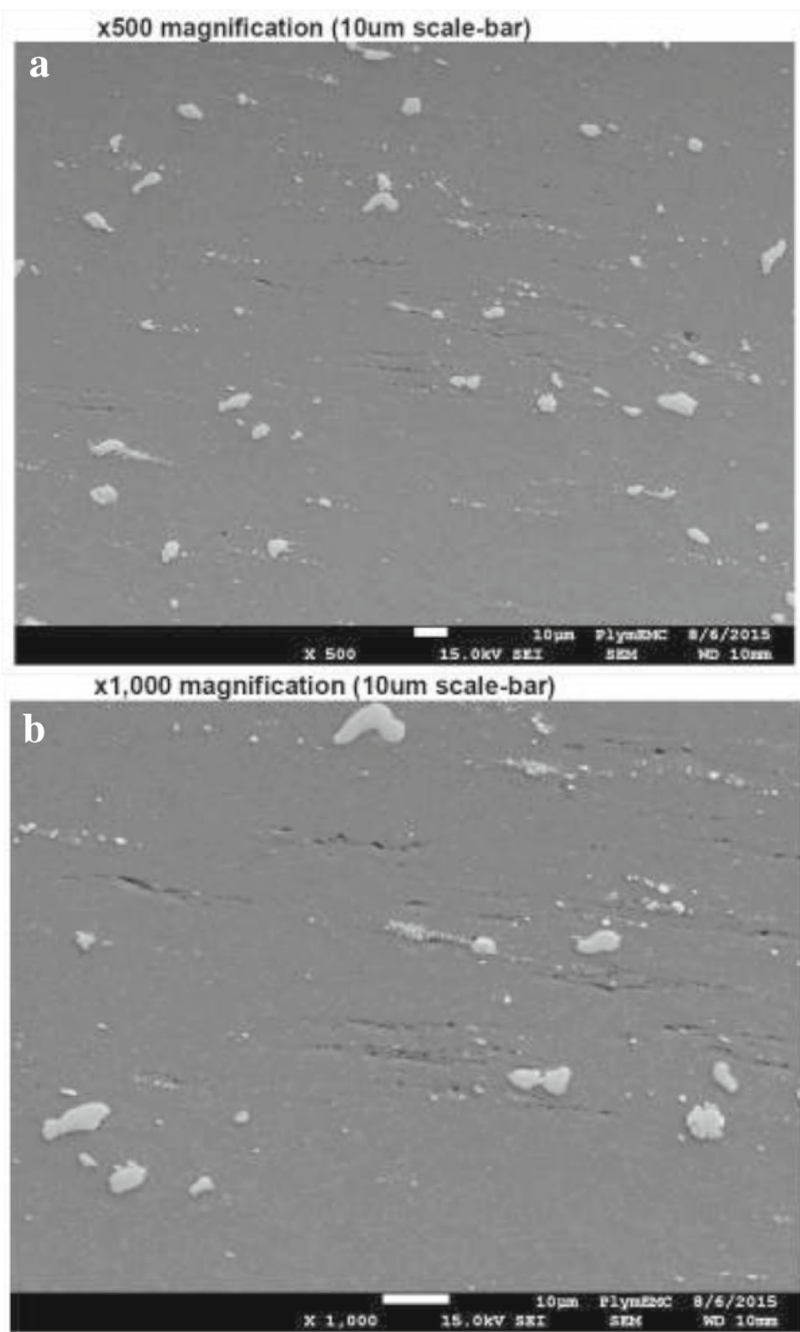

$x 3,500$ magnification (1um scale-bar)

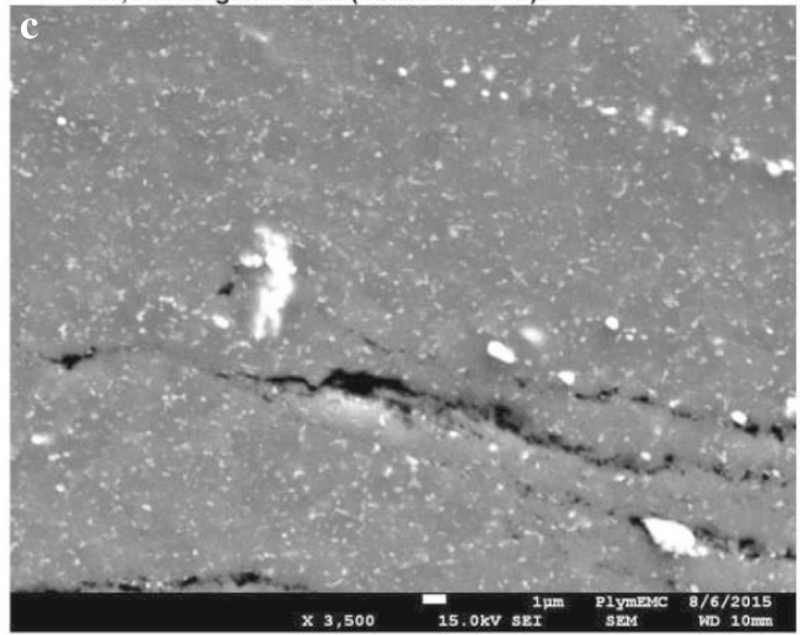

Fig. 11 SEM micrographs of a subsample of FSW Sample 2 with a high level of nonlinear signature, shown using three different magnification levels, illustrating the presence of micro-cracks in the nugget zone: magnifications $\mathbf{a} \times 500, \mathbf{b} \times 1000, \mathbf{c} \times 3500$, after [30] 
in Figs. 8 and 9, the pulse-inversion chirp-coded weld harmonic imaging (PI-CWHI) produces a $2 \mathrm{D}$ heat map of the presence of nonlinearity along the weld centerline. Horizontal and vertical axes on the $2 \mathrm{D}$ heat map represent the frequency and the measurement position along the weld centerline respectively. The colors correspond to the intensity of the normalized pulse-inversion spectrum.

The results for Sample 1 are shown in Fig. 8a, and exhibit a high degree of nonlinearity for positions ranging from 150 to $350 \mathrm{~mm}$, indicating that the severity of micro-crack features is dominant in this zone. In fact, in practical FSW inspection using nonlinear wave analysis, two sources may contribute to the observed nonlinearity: the micro-crack density and the instrumentation. Since the instrumentation does not vary along the weld during the scanning, we can be sure that the micro-crack density is the main contributor to the PI-CWHI technique at the increased nonlinear harmonic signature zones. The nonlinearity is thus generated and accumulated during the acoustic wave propagation through the weld zone. Repeated tests on the same sample also eliminate the potential influence of bad coupling on the data analysis when moving along the weld. The Damage Index calculated from the spectra in the heat map of Fig. 8a are shown in Fig. 8b. The ratio between the highest and lowest Damage Index along the weld center line is 1.6.

As a second illustration, Fig. 9a shows the pulse-inversion spectral image for a similar 7XXX aluminum alloy sample: Sample 2. Here, the first part of the weld can be easily discerned as a severe defect zone, ranging from 0 to $150 \mathrm{~mm}$. A more mild damage zone is found around $350 \mathrm{~mm}$. Figure $9 \mathrm{~b}$ show that the ratio between the highest and lowest Damage Index along the weld center line for this sample is 2.2.

It is important to note that, for practical use in FSW inspection, the color scale of the 2D heat maps should be adjusted to a tolerable level of nonlinearity which is linked to an acceptable quality level of micro-crack density. For instance, zones containing a micro-crack density below $0.15 \%$ may be considered as acceptable for FSW. Therefore, the lower value of the color scale should be set to a value representing crack density zones greater than $0.15 \%$. Of course this requires a prior validation of the technique and an a-priori known correlation between the observed nonlinearity and the actual micro-crack density inside similar calibrated samples.

The validation of the PI-CWHI technique and a preliminary effort to establish a correlation between the measured nonlinearity and the actual micro-crack density was carried out using optical microscopy and scanning electron microscopy (SEM) analysis on three subsamples extracted from different regions of Sample 2, corresponding to zones with low (DI=60, subsample 2.1), average ( $D I=90$, subsample 2.2) and high harmonic (DI=120, subsample 2.3) levels [30]. The positions of these subsamples in the scan along the weldline are indicated on Fig. 9. The optical microscopy images (obtained by way of an Olympus U-TV0.5xC-3 camera) and a quantitative analysis of these images reveals that the level of micro-crack density is substantially different between these three samples, see table 1. Figure 10 indeed shows qualitatively that the micro-crack density is increasing when the nonlinearity signature is rising. Note that in Fig. 10a, the apparent quasi-horizontal line near the bottom does not represent a crack, but is the unfortunate residual of a cleaning operation using acetone. In addition, in order to measure the length and quantity of the micro-cracks, SEM was carried out, as illustrated in Fig. 11 for the subsample 2.3 with the highest nonlinearity signature. Between the subsamples with the lowest and the highest nonlinearities, corresponding to a twofold increase in the nonlinear signature, a tenfold change from 0.15 to $1.5 \%$ in crack density (percentage damage per unit area) was observed. These findings are in good agreement with a previously conducted experimental study carried out by Van Den Abeele et al. on CFRP composite samples, where a proportional relationship was found between the micro-crack density and the nonlinearity signature in CFRP composite samples after being exposed to increasing thermal loading [31].

\section{Conclusion}

Pulse-inversion chirp-coded weld harmonic imaging (PICWHI) is a powerful technique for identifying sites of mild and severe micro-crack density in the root part of the nugget zone of friction stir welded joints. The technique exploits and quantifies the nonlinear distortion of the transmitted coded chirp excitation within the friction stir weld zone. A chirpcoded excitation signal is transmitted, using successively a positive and a negative polarity, and the back-wall reflected signals containing the fundamental and harmonic frequencies are subsequently decoded via pulse-inversion. By stacking the normalized pulse inversion spectra, a heat map of the nonlinearity signature can be obtained along the weld centerline. Quantification of micro-cracks in the experimental validation study showed that the crack density is increasing with the nonlinear signature, allowing an easy interpretation of the heat maps in terms of damage.

Future work will focus on the optimization of chirpcoded excitation in terms of fractional bandwidth and time duration. The basic concept of the proposed pulse-inversion chirp-coded nonlinear technique can be transferred and implemented in phased array (PA) systems in order to benefit from full matrix capture (FMC) and total focusing method (TFM) for harmonic imaging of the entire weld volume of the nugget zone rather than solely inspecting the root part of the weld. 
Acknowledgements The research leading to these results has gratefully received funding from the European Union Seventh Framework Program (FP7/2007-2013) for research, technological development and demonstration under the Grant Agreements No. 315435 (StirScan) and No. 314768 (ALAMSA). Elements of this work are subject to patent applications on behalf of Theta Technologies Limited.

\section{References}

1. Mishra, R.S., Ma, Z.Y.: Friction stir welding and processing. Mater. Sci. Eng. R. 50, 1-78 (2005)

2. Nandan, R., Debroy, T., Bhadeshia, H.: Recent advances in frictionstir welding-process, weldment structure and properties. Prog. Mater. Sci. 53, 980-1023 (2008)

3. Sato, Y.S., Takauchi, H., Park, S.H.C., Kokawa, H.: Characteristics of the kissing-bond in friction stir welded $\mathrm{Al}$ alloy. Mater. Sci. Eng. A. 405(2005), 333-338 (1050)

4. Tabatabaeipour, M., Hettler, J., Delrue, S., Van Den Abeele, K.: Non-destructive ultrasonic examination of root defects in friction stir welded butt-joints. NDT E Int. 80, 23-34 (2016)

5. Dai, Q.L., Wang, X.Y., Hou, Z.G., Wu, J.J., Shi, Q.Y.: Microcavities accompanying a zigzag line in friction stir welded A6082-T6 alloy joint. Sci. Technol. Weld. Join. 20, 68-74 (2015)

6. Oosterkamp, A., Osterkamp, L.D., Nordeide, A.: Kissing bond phenomena in solid-state welds of aluminum alloys. Weld. J. 83(8), 225-231 (2004)

7. Kadlec, M., Ružek, R., Nováková, L.: Mechanical behaviour of AA 7475 friction stir welds with the kissing bond defect. Int. J. Fatigue 74, 7-19 (2014)

8. Goldfine, N., Ziberstein, V., Schlicker, D.E., Grundy, D.C., Shay, I., Washabaugh, A.P.: High resolution inductive sensor arrays for material and defect characterization of welds. US Patent No. $6,727,691,2004$

9. Goldfine, N., Grundy, D., Ziberstein, V.: Friction stir weld inspection through conductivity using shaped field MWM-arrays. In: 6th International Conference on Trends in Welding. ASM Int., Callaway Garden, GA, USA (2003)

10. Ohara, Y., Yamamoto, S., Mihara, T., Yamanaka, K.: Ultrasonic evaluation of closed cracks using subharmonic phased array. Jpn. J. Appl. Phys. 47, 3908-3915 (2008)

11. Van Den Abeele, K.E., Sutin, A., Carmeliet, J., Johnson, P.A.: Micro-damage diagnostics using nonlinear elastic wave spectroscopy (NEWS). NDT E Int. 34, 239-248 (2001)

12. Scalerandi, M.: Power laws and elastic nonlinearity in materials with complex microstructure. Phys. Lett. A. 1, 1-9 (2015). doi:10. 1016/j.physleta.2015.11.004

13. Van Den Abeele, K., Carmeliet, J., Ten Cate, J.A., Johnson, P.A.: Nonlinear elastic wave spectroscopy (NEWS) techniques to discern material damage, Part II: single-mode nonlinear resonance acoustic spectroscopy. J. Res. Nondestruct. Eval. 12, 31-42 (2000)

14. Hwang, J.-J., Simpson, D.H.: Two pulse technique for ultrasonic harmonic imaging. US Patent No. 5,951,478, 1999

15. Chapman, C.S., Lazenby, J.C.: Ultrasound imaging system employing phase inversion subtraction to enhance the image. US Patent No 5,632,27, 1997
16. Ma, Q., Ma, Y., Gong, X., Zhang, D.: Improvement of tissue harmonic imaging using the pulse-inversion technique. Ultrasound Med. Biol. 31, 889-94 (2005)

17. Simpson, D.H., Chin, C.T., Burns, P.N.: Pulse inversion Doppler: a new method for detecting nonlinear echoes from microbubble contrast agents. IEEE Trans. Ultrason. Ferroelectr. Freq. Control. 46, 372-82 (1999)

18. Dos Santos, S., Vejvodova, S., Prevorovsky, Z.: Nonlinear signal processing for ultrasonic imaging of material complexity. Proc. Est. Acad. Sci. 59, 108 (2010)

19. Armitage, P.R., Wright, C.D.: Design, development and testing of multi-functional non-linear ultrasonic instrumentation for the detection of defects and damage in CFRP materials and structures. Compos. Sci. Technol. 87, 149-156 (2013)

20. Shen, C.-C., Chou, Y.-H., Li, P.-C.: Pulse inversion techniques in ultrasonic nonlinear imaging. J. Med. Ultrasound. 13, 3-17 (2005). doi:10.1016/S0929-6441(09)60073-4

21. O'Donnell, M.: Coded excitation system for improving the imaging systems. IEEE Trans. Ultrason. Ferroelectr. Freq. Control. 39, 341351 (1992). doi:10.1109/58.143168

22. Chiao, R.Y., Hao, X.: Coded excitation for diagnostic ultrasound: a system developer's perspective. IEEE Trans. Ultrason. Ferroelectr. Freq. Control. 52, 160-170 (2005)

23. Shekhar, H., Huntzicker, S., Awuor, I., Doyley, M.M.: Chirp-coded ultraharmonic imaging with a modified clinical intravascular ultrasound system. Ultrason. Imaging 38(6), 403-419 (2015)

24. Shekhar, H., Doyley, M.M.: The response of phospholipidencapsulated microbubbles to chirp-coded excitation: implications for high-frequency nonlinear imaging. J. Acoust. Soc. Am. 133, 3145-58 (2013)

25. Song, J., Kim, S., Sohn, H.Y., Song, T.K., Yoo, Y.M.: Coded excitation for ultrasound tissue harmonic imaging. Ultrasonics $\mathbf{5 0}$, 613-619 (2010)

26. Park, J., Huang, Y., Chen, R., Lee, J., Cummins, T.M., Zhou, Q., Lien, C.L., Shung, K.K.: Pulse inversion chirp coded tissue harmonic imaging (PI-CTHI) of zebrafish heart using high frame rate ultrasound biomicroscopy. Ann. Biomed. Eng. 41, 41-52 (2013)

27. Solodov, I.: Ultrasonic resonance of defects for nonlinear acoustic imaging and NDT. In: 11th European Conference on NonDestructive Testing (ECNDT). Prague, Czech Republic, 2014

28. Solodov, I., Rahammer, M., Gulnizkij, N.: Highly-sensitive and frequency-selective imaging of defects via local defect resonance. In: 11th European Conference on Non-Destructive Testing (ECNDT). Prague, Czech Republic, 2014

29. Matlack, K.H., Kim, J.Y., Jacobs, L.J., Qu, J.: Review of scond harmonic generation measurement techniques for material state determination in metals. J. Nondestruct. Eval. 34(1), 273 (2015)

30. Sewell, N.T., Wright, J.R., Wright, J.C.S.: Detection of kissing bonds in friction stir welds. In: 11th International Symposium on Friction Stir Welding, 2016

31. Van Den Abeele, K., Le Bas, P.Y., Van Damme, B., Katkowski, T.: Quantification of material nonlinearity in relation to microdamage density using nonlinear reverberation spectroscopy: experimental and theoretical study. J. Acoust. Soc. Am. 126, 963-72 (2009) 